\title{
Iterated egalitarian compromise solution to bargaining problems and midpoint domination
}

\author{
Emin Karagözoğlu ${ }^{\text {a,b,* }}$, Elif Özcan Tok ${ }^{\mathrm{a}, \mathrm{c}}$ \\ a Bilkent University, Turkey \\ b CESifo, Munich, Germany \\ c CBRT, Turkey
}

\section{A R T I C L E I N F O}

\section{Article history:}

Received 27 November 2017

Received in revised form 12 February 2018

Accepted 19 February 2018

Available online 6 March 2018

\section{Keywords:}

Axioms

Bargaining problem

Egalitarianism

Midpoint domination

Randomized dictatorship

\begin{abstract}
A B S T R A C T
We introduce a new solution for two-person bargaining problems: the iterated egalitarian compromise solution. It is defined by using two prominent bargaining solutions, the egalitarian solution (Kalai, 1977) and the equal-loss solution (Chun, 1988), in an iterative fashion. While neither of these two solutions satisfy midpoint domination - an appealing normative property - we show that the iterated egalitarian compromise solution does so.
\end{abstract}

(c) 2018 Elsevier B.V. All rights reserved.

\section{Introduction}

In a seminal paper, Nash (1950) [7] introduced the axiomatic treatment of bargaining problems. Over the last seven decades, the axiomatic approach has attracted a considerable attention from researchers studying bargaining (see [6] for an overview). The axiomatic literature on bargaining has been productive in coming up with solution concepts with appealing normative properties. Two prominent solutions of interest for the current paper are the egalitarian solution ( $E$, for short) due to Kalai (1977) [3] and the equal loss solution (EL, for short) due to Chun (1988) [2]. As their names suggest, both solutions apply an egalitarian notion of justice in proposing outcomes to bargaining problems. More precisely, for each bargaining problem, $E$ proposes the maximum utility profile that gives each agent an equal gain over his disagreement outcome, whereas $E L$ proposes the maximum utility profile that gives each agent an equal loss over his ideal outcome (i.e., the best possible outcome for the agent among the outcomes that are individually rational for both).

These two solutions share a common weakness: both of them fail to satisfy a basic yet desirable normative requirement that a solution should assign each agent at least half of his ideal point outcome in all bargaining problems. It can be rephrased as, for any problem, an outcome proposed by a solution should be Pareto

\footnotetext{
* Correspondence to: Department of Economics, Bilkent University, 06800 Bilkent, Ankara, Turkey.

E-mail address: karagozoglu@bilkent.edu.tr (E. Karagözoğlu).
}

superior to the randomized dictatorship outcome. This requirement was introduced in [11] and known as midpoint domination $(M D$, for short). As Rachmilevitch (2017) [9] points out, midpoint domination has both fairness and efficiency connotations. On one hand, it requires both agents to receive at least half of their ideal point outcomes (fairness) and on the other, it requires the proposed outcome to be Pareto superior to the midpoint (efficiency). Hence, it is an appealing normative property.

In this paper, we, first, introduce a new solution concept for two-person bargaining problems: iterated egalitarian compromise solution (IEC, for short). For a problem where $E$ and $E L$ propose the same outcome, the outcome proposed by IEC coincides with theirs. For a problem where $E$ and $E L$ propose different outcomes, IEC proposes a compromise in an iterative fashion, by using the proposed outcomes of $E$ and $E L$ at each iteration step. Hence, the name, iterated egalitarian compromise. Second, we show that IEC is well-defined, i.e. for any problem in the domain of two-person bargaining problems we consider, it proposes a unique outcome, defined as the limit of an iterative process. Finally, we show that IEC satisfies midpoint domination despite the fact that neither of the solutions it is based on does so, a fact that makes the result a nontrivial one. A recent attempt in a similar direction is [9]. The author proposes a midpoint-robust (i.e., satisfying midpoint domination) version of $E$.

The paper is organized as follows: in Section 2, we introduce the bargaining problem, define the solutions of interest, and the midpoint domination property. In Section 3, we prove that IEC is well-defined and it satisfies midpoint domination. Furthermore, 
we relate IEC to another prominent solution concept that has an egalitarian flavor, the Kalai-Smorodinsky solution [4]. Section 4 concludes with final remarks.

\section{The model}

A simple two-person bargaining problem is denoted by $S \subset$ $\mathbb{R}^{2}$. It satisfies the following properties: it is (i) non-empty, (ii) closed, and bounded from above, (iii) convex, (iv) comprehensive, (v) $S \cap \mathbb{R}_{++}^{2} \neq \varnothing$, and (vi) it contains the disagreement outcome, $\mathbf{0} \equiv(0,0)$. The axiomatic properties of the solutions we will use allow us to normalize the disagreement outcome to $(0,0)$. Since we will do that in what follows, we denote the problem by $S$ instead of $(S, d)$. Intuitively, $S$ represents all the utility vectors that can be achieved by the agents. The non-emptiness is to make the problem non-trivial. The closedness of $S$ means that the set of physical agreements is closed and that agents' payoff functions are continuous. The boundedness from above means that the maximum utility an agent can achieve out of an agreement is finite. The convexity assumption means that agents could agree to take a coin-toss between two outcomes and that each agent's payoff from the coin toss is the average of his/her payoffs from these outcomes. Comprehensiveness stipulates that utility is freely disposable down to the disagreement utilities. $S \cap \mathbb{R}_{++}^{2} \neq \emptyset$ rules out degenerate problems where no agreement can make all agents better off than the disagreement outcome. Finally, $\mathbf{0} \in S$ means that the agents can agree to disagree. We denote the set of all such problems by $\Sigma$. For every $S \subset \mathbb{R}^{2}$, its weak (strong) Pareto optimal set is defined as $W P O(S) \equiv\{y \in S \mid x>y$ implies $x \notin S\}(P O(S) \equiv$ $\{y \in S \mid x \supsetneqq y$ implies $x \notin S\})$. Here, we will focus on a subdomain of $\Sigma$, denoted by $\widehat{\Sigma}$, whose weak and strong Pareto frontiers coincide (i.e., the bargaining frontier does not have any horizontal or vertical segments). The importance of this assumption will be explained later in the proof of Proposition 1. Finally, a bargaining solution $F$ is a function, which assigns to any bargaining problem $S$, a unique point in it.

The egalitarian solution [3] equalizes agents' gains over their disagreement outcomes. Accordingly, it assigns to each $S$ the point, $E(S)$ with identical $(x, y)$-coordinates and $E(S)$ is the maximum possible. This corresponds to selecting the intersection point of the Pareto frontier and the 45-degree line drawn from the disagreement point (in our case, the origin). The equal loss solution [2] equalizes agents' losses from their ideal point outcomes. Formally, ideal point, introduced by [4], is defined as $a_{i}(S) \equiv \max \left\{s_{i}: s \in S\right\}$, where $a_{i}(s)$ denotes agent $i$ 's ideal point outcome. Accordingly, the equal loss solution assigns to each $S$, the point $E L(S)=a(S)-$ $(l, l)$, where $l$ is the minimum possible. This corresponds to selecting the point at the intersection of the Pareto frontier and the 45-degree line drawn from the ideal point. Note that for all $S \subset \Sigma$, if $a_{1}(S)>a_{2}(S)$, then $E L_{1}(S)>E_{1}(S)$ and $E_{2}(S)>E L_{2}(S)$, and vicea-versa.

A solution $F$ satisfies midpoint domination, if it proposes an outcome $F(S) \geq m p(S) \equiv \frac{1}{2} a(S)$, for all $S$. Fig. 1 shows an example, where both $E$ and $E L$ violate $M D$. Note that the bargaining problem in the example is in $\widehat{\Sigma}$.

The iterated egalitarian compromise solution (or IEC, for short) assigns to each $S \in \widehat{\Sigma}$, the point $x$, if $E(S)=E L(S)=$ $x$ and assigns the point $y \equiv \cap_{t \in \mathbb{N}} P O\left(S_{t}\right)$, where $S_{0} \equiv S$ and the bargaining problem in iteration step $t, S_{t}$, for $t \geq 1$ is derived by applying $E$ and $E L$ to $S_{t-1}$ in a way that, the origin (i.e., the disagreement point) of $S_{t}$ denoted by $o\left(S_{t}\right)$, is $o\left(S_{t}\right)=\left(\min \left\{E_{1}\left(S_{t-1}\right), E L_{1}\left(S_{t-1}\right)\right\}, \min \left\{E_{2}\left(S_{t-1}\right), E L_{2}\left(S_{t-1}\right)\right\}\right)$ and consequently $a\left(S_{t}\right)=\left(\max \left\{E_{1}\left(S_{t-1}\right), E L_{1}\left(S_{t-1}\right)\right\}, \max \left\{E_{2}\left(S_{t-1}\right)\right.\right.$, $\left.\left.E L_{2}\left(S_{t-1}\right)\right\}\right)$.

IEC could be interpreted as a conflict resolution mechanism, which resolves the conflict between $E$ and $E L$ in a step-by-step

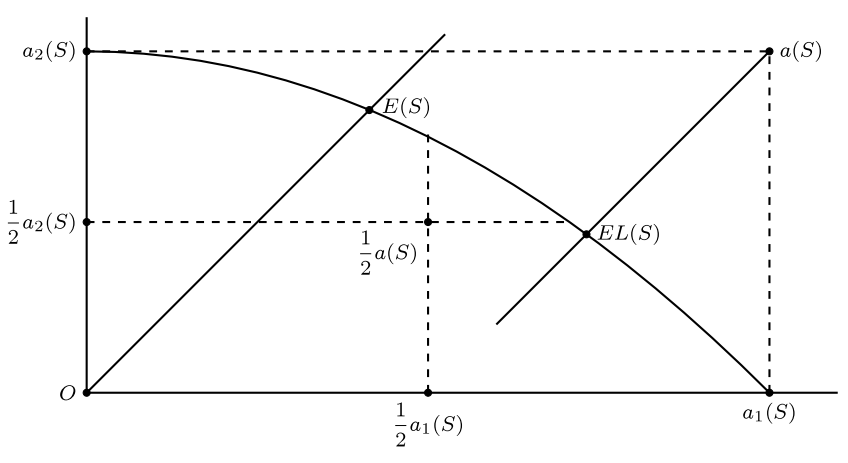

Fig. 1. $E$ and $E L$ violate $M D$.

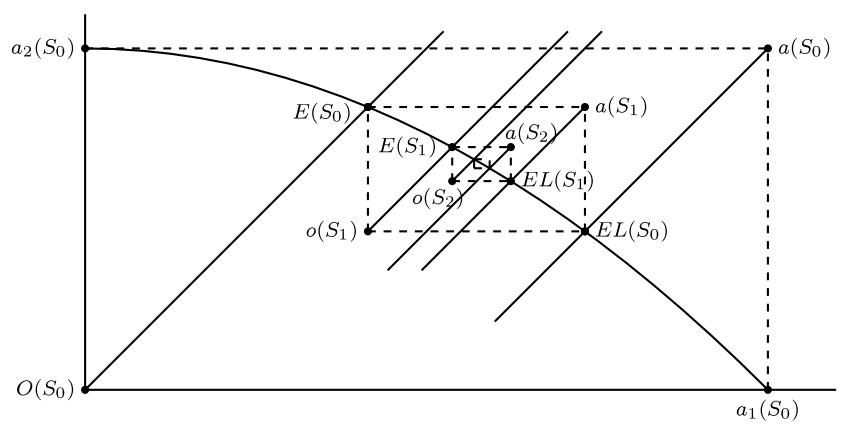

Fig. 2. Iterated egalitarian compromise solution.

fashion, by using the minimal outcomes in each iteration as starting points and the maximal outcomes as ideals for the bargaining problem in the next step. Fig. 2 shows how IEC operates in a problem where $E$ and $E L$ propose different outcomes.

\section{The result}

First, we prove that $I E C$ is well-defined, i.e. for all $S \in \widehat{\Sigma}$ the iterative process embedded in IEC converges to a single point.

Proposition 1. For all $S \in \widehat{\Sigma}$, IEC is well-defined.

Proof. First, consider a symmetric bargaining problem, $S \equiv S_{0}$. In this case, IEC proposes a single outcome, since $E\left(S_{0}\right)=E L\left(S_{0}\right)$. Now, consider an asymmetric problem, $S \equiv S_{0} \in \widehat{\Sigma}$. Without loss of generality, suppose that $a_{1}\left(S_{0}\right)>a_{2}\left(S_{0}\right)$. For notational convenience, let $a_{1}\left(S_{t}\right)-o_{1}\left(S_{t}\right)=\alpha_{t}$ and $a_{2}\left(S_{t}\right)-o_{2}\left(S_{t}\right)=\beta_{t}$. Since both $E$ and $E L$ operate via upward-sloping 45 -degree lines, for each iteration step $t$, we get $\alpha_{t+1}+\beta_{t+1}=\left|\alpha_{t}-\beta_{t}\right|$. The sequences $\left(\alpha_{t}\right)$ and $\left(\beta_{t}\right)$ are decreasing and bounded below $\left(\alpha_{t} \geq 0, \beta_{t} \geq 0\right)$. Thus, there exist some $\bar{\alpha}$ and $\bar{\beta}$ such that $\lim _{t \rightarrow \infty} \alpha_{t_{-}}=\bar{\alpha} \geq 0$ and $\lim _{t \rightarrow \infty} \beta_{t}=\bar{\beta} \geq 0$. As $t \rightarrow \infty$, we have $\bar{\alpha}+\bar{\beta}=|\bar{\alpha}-\bar{\beta}|$, which requires at least one of $\bar{\alpha}$ and $\bar{\beta}$ to be equal to zero. Suppose without loss of generality that $\bar{\alpha}=0$. Since bargaining frontier has no horizontal or vertical segments, $\bar{\beta}=0$ as well, which implies that our iteration algorithm converges to a single point (i.e., IEC is single valued).

Note that beyond showing that the solution is well-defined, this result provides a useful insight: the convergence of the iterative process can be interpreted as an explicit convergence of interests between $E$ and $E L$. In addition to this, Proposition 2 will establish the fact that this convergence of interests satisfies an appealing property. 


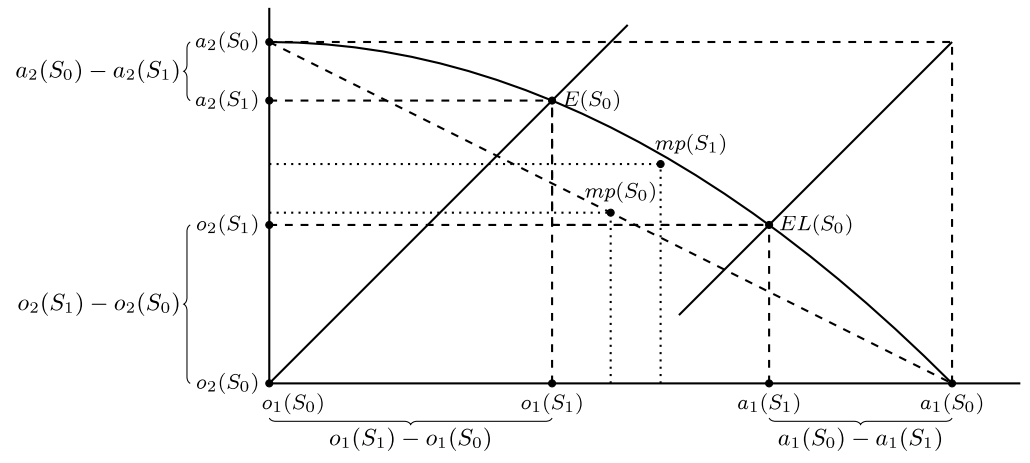

Fig. 3. Changes in the midpoints (nonlinear, asymmetric case).

Remark 1. For some iteration step $t^{\prime}$, the relative positions of $E$ and $E L$ on the frontier may change, i.e. $a_{2}\left(S_{t^{\prime}}\right)>a_{1}\left(S_{t^{\prime}}\right)$. Nevertheless, by the definitions of disagreement point and ideal point, and the way our iteration mechanism operates, $\alpha_{t^{\prime}} \geq 0, \beta_{t^{\prime}} \geq 0$, and these sequences continue to decrease.

Remark 2. The domain restriction we made (i.e., bargaining frontier has no horizontal/vertical segments) is necessary for the argument in last step of the proof (of Proposition 1) to be valid. If the Pareto frontier had horizontal/vertical segments, iterative process may converge to a set that has more than one element.

The following corollary shows the relationship between $\operatorname{IEC}\left(S_{t}\right)$ and $m p\left(S_{t}\right)$ in the limit as $t \rightarrow \infty$, and it will be utilized in the proof of Proposition 2.

Corollary 1. For all $S \in \widehat{\Sigma}, \lim _{t \rightarrow \infty} \operatorname{IEC}\left(S_{t}\right)=\lim _{t \rightarrow \infty} m p\left(S_{t}\right)$.

Proof. The proof directly follows from the following facts: (i) IEC is single-valued, (ii) the corresponding bargaining problem collapses to a point in the limit, and (iii) the midpoint of a single point is itself.

Now, we are ready to state our main result.

Proposition 2. For all $S \in \widehat{\Sigma}$, IEC satisfies MD.

Proof. We will prove this statement in two steps. To do that, we partition $\widehat{\Sigma}$ into two subsets: (i) problems with linear bargaining frontiers $\left(\widehat{\Sigma}_{\text {lin }}\right)$, (ii) problems with non-linear bargaining frontiers $\left(\widehat{\Sigma}_{\text {nlin }}\right)$. Below, we will show that IEC satisfies MD in both subsets.

Claim 1. For all $S \in \widehat{\Sigma}_{\text {lin }}$, IEC satisfies MD.

Proof. If $S \equiv S_{0}$ is symmetric, i.e., $a_{1}\left(S_{0}\right)=a_{2}\left(S_{0}\right)$, then trivially $\operatorname{IEC}\left(S_{0}\right)=E\left(S_{0}\right)=E L\left(S_{0}\right)=x$, where $x=m p\left(S_{0}\right)$. Suppose now that $S \equiv S_{0}$ is asymmetric, i.e., $a_{1}\left(S_{0}\right) \neq a_{2}\left(S_{0}\right)$. Furthermore, without loss of generality, assume that $a_{1}\left(S_{0}\right)>a_{2}\left(S_{0}\right)$. Then, $E\left(S_{0}\right) \neq E L\left(S_{0}\right)$. The linearity of the Pareto frontier implies that the segments of the frontier cut by $E$ and $E L$ (from two ends) in each iteration step are of equal length. Formally, for all $t>0$, $o_{1}\left(S_{t}\right)-o_{1}\left(S_{t-1}\right)=a_{1}\left(S_{t-1}\right)-a_{1}\left(S_{t}\right)$ and $o_{2}\left(S_{t}\right)-o_{2}\left(S_{t-1}\right)=$ $a_{2}\left(S_{t-1}\right)-a_{2}\left(S_{t}\right)$. Therefore, $m p\left(S_{0}\right)=m p\left(S_{t}\right)$, for all $t>0$. Proposition 1 and Corollary 1 imply that $\operatorname{IEC}(S)=m p(S)$. Hence, the result follows.

Claim 2. For all $S \in \widehat{\Sigma}_{\text {nlin }}$, IEC satisfies MD.

Proof. If $S \equiv S_{0}$ is symmetric, i.e., $a_{1}\left(S_{0}\right)=a_{2}\left(S_{0}\right)$, then trivially $\operatorname{IEC}\left(S_{0}\right)=E\left(S_{0}\right)=E L\left(S_{0}\right)=x$, where $x \geq m p\left(S_{0}\right)$. Suppose now that $S \equiv S_{0}$ is asymmetric, i.e., $a_{1}\left(S_{0}\right) \neq a_{2}\left(S_{0}\right)$. Furthermore, without loss of generality, assume that $a_{1}\left(S_{0}\right)>a_{2}\left(S_{0}\right)$. Then, $E\left(S_{0}\right) \neq E L\left(S_{0}\right)$. The convexity of $S_{0}$ and the non-linearity of the bargaining frontier imply that $o_{1}\left(S_{t}\right)-o_{1}\left(S_{t-1}\right) \geq a_{1}\left(S_{t-1}\right)-a_{1}\left(S_{t}\right)$ and $o_{2}\left(S_{t}\right)-o_{2}\left(S_{t-1}\right) \geq a_{2}\left(S_{t-1}\right)-a_{2}\left(S_{t}\right)$ for all $t \geq 1$ and these inequalities strictly hold for some $t$. But this implies that $m p\left(S_{t}\right) \gg m p\left(S_{t-1}\right)$ for all $t>0$ (i.e., each iteration step moves the midpoint in the north-east direction). So, $\lim _{t \rightarrow \infty} m p\left(S_{t}\right) \gg m p\left(S_{0}\right)$ (see Fig. 3). From Corollary 1, we know that $y \equiv \lim _{t \rightarrow \infty} \operatorname{IEC}\left(S_{t}\right)=$ $\lim _{t \rightarrow \infty} m p\left(S_{t}\right)$. Therefore, $\operatorname{IEC}(S) \gg m p(S)$. The same result is valid for the case of $a_{1}\left(S_{0}\right)<a_{2}\left(S_{0}\right)$, as well. Hence, the result follows.

Kalai-Smorodinsky solution (KS for short) also satisfies $M D$, and like $E$ and $E L$, it utilizes an egalitarian justice norm ( $K S$ equalizes the ratios of maximal gains across players). As such it can be thought as another alternative, but it rules out inter-personal utility comparisons whereas $I E C$, like $E$ and $E L$, is built on the premise that such comparisons are possible. A direct implication of Proposition 2 on the relationship between IEC and $K S$ is given in the following corollary.

Corollary 2. For all $S \in \widehat{\Sigma}_{\text {lin }}, \operatorname{IEC}(S)=K S(S)$.

Proof. The proof directly follows from the following facts: in a bargaining problem $S \in \widehat{\Sigma}_{\text {lin }}$, (i) midpoint is on the $P O(S)$, and thus the only way for a solution to satisfy $M D$ is to propose the midpoint, (ii) $K S$ proposes the midpoint, and (iii) IEC satisfies $M D$ in $\widehat{\Sigma}_{\text {lin }}$ (from Claim 1 in Proposition 2).

Note that this statement is not necessarily true for $S \in \widehat{\Sigma}_{\text {nlin }}$ (see Fig. 4). Furthermore, it is neither valid for $E$ nor for $E L$, even in $\widehat{\Sigma}_{\text {lin }}$.

It is worth mentioning here that $E$ and $E L$ are duals of each other. Recognizing this fact, one can draw another similarity between IEC and KS. Recently, Karagözoğlu and Rachmilevitch (2017) [5], in a paper where they provided three new characterizations of $K S$, showed that the outcome proposed by $K S$ always lies (i.e., sandwiched) in between the outcomes proposed by two other solutions with egalitarian objectives: the equal area solution ( $E A$ for short) and the dual of the equal area solution (DEA for short). Along similar lines, the outcome proposed by IEC, by construction, always lies in between the outcomes proposed by $E$ and $E L$, again, two egalitarian solutions that are duals of each other. Reader is referred to [1] for $E A$, [5] for $D E A$, and Lemma 2 in [5] for the abovementioned "sandwich" result.

\section{Conclusion}

We introduced a new solution concept, $I E C$, for two-person bargaining problems, which is based on two well-known egalitarian solution concepts, $E$ and EL. IEC mimics a conflict resolution 


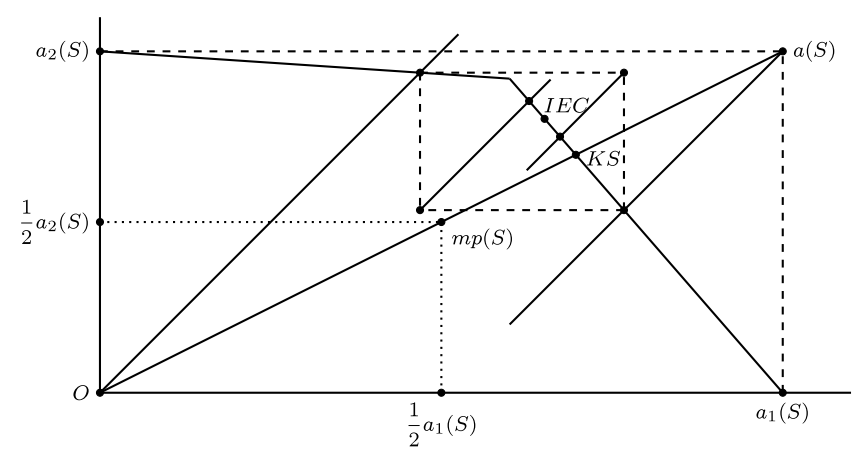

Fig. 4. The relation between KS and IEC.

mechanism and satisfies an appealing normative property, midpoint domination, which is violated by both $E$ and $E L$. Thus, IEC is a reasonable alternative, especially if one wants to (i) utilize an egalitarian justice norm in problems where $E$ and $E L$ disagree and (ii) operate in a domain that allows inter-personal utility comparisons.

Our results lead to some new questions. Below, we describe three of them.

(1) In addition to $M D$, IEC satisfies Pareto optimality, symmetry, and scale invariance [7], by definition. Furthermore, the proof of Claim 2 in Proposition 2 implies that it satisfies restricted monotonicity [10], as well. It would be of interest, from a normative perspective, to study which axiomatic properties would characterize IEC.

(2) As we argued in Section 3, there are certain similarities between IEC and KS, in that both are sandwiched between two egalitarian solutions, which are duals of each other: IEC is sandwiched by $E$ and $E L$, whereas $K S$ is sandwiched by $E A$ and $D E A$. Further investigation of the relationships between these six solutions with egalitarian objectives would be of interest.

(3) Finally, the iterative process IEC utilizes resembles the stepby-step nature of negotiations. Thus, whether a strategic founda- tion for IEC can be provided is an interesting question, in the spirit of the Nash program [8].

These are all beyond the scope of this paper, and hence left for future research.

\section{Acknowledgments}

The authors thank an anonymous reviewer for constructive comments, which improved the paper. They also thank Serhat Doğan, Aurelian Gheondea, Tarık Kara, Selman Erol, and Shiran Rachmilevitch for fruitful discussions and valuable comments. Emin Karagözoğlu thanks TÜBITAK (The Scientific and Technological Research Council of Turkey) for the post-doctoral research fellowship, and Massachusetts Institute of Technology, Department of Economics for their hospitality. Usual disclaimers apply.

\section{References}

[1] N. Anbarc1, J.P. Bigelow, The area monotonic solution to the cooperative bargaining problem, Math. Social Sci. 28 (1994) 133-142.

[2] Y. Chun, The equal-loss principle for bargaining problems, Econom. Lett. 26 (1988) 103-106.

[3] E. Kalai, Proportional solutions to bargaining situations: interpersonal utility comparisons, Econometrica 45 (1977) 1623-1630.

[4] E. Kalai, M. Smorodinsky, Other solutions to Nash's bargaining problem, Econometrica 43 (1975) 513-518.

[5] E. Karagözoğlu, S. Rachmilevitch, Duality, area-considerations, and the KalaiSmorodinsky Solution, Oper. Res. Lett. 45 (2017) 30-33.

[6] Ö. Kıbris, Cooperative game theory approaches to negotiation, in: D.M. Kilgour, C. Eden (Eds.), Handbook of Group Decision and Negotiation, Vol. 4, Springer, The Netherlands, 2010, pp. 151-166.

[7] J.F. Nash, The bargaining problem, Econometrica 18 (1950) 155-162.

[8] J.F. Nash, Two-person cooperative games, Econometrica 21 (1953) 128-140.

[9] S. Rachmilevitch, A midpoint robust egalitarian bargaining solution, Working paper, 2017.

[10] A.E. Roth, An impossibility result concerning n-person bargaining games, Internat. J. Game Theory 8 (1979) 129-132.

[11] J. Sobel, Distortion of utilities and the bargaining problem, Econometrica 49 (1981) 597-619. 